\title{
Survey on Two-Factor Authentication with Security in Online Transaction
}

\author{
Shilpa R*, Janhavi V \\ Department of Computer Science, Vidyavardhaka College of Engineering, Mysuru \\ DOI: https://doi.org/10.21467/proceedings.1.66 \\ * Corresponding author email: shilparamesh0@gmail.com
}

\begin{abstract}
The most well known two-factor authentication system, online exchange password, MPIN verification has been a subject of concentrated research in the previous two decades, and several this kind of plans have been proposed. In the majority of these investigations, there is no exhaustive and systematical metric accessible for plans to be evaluated impartially, and the creators give new plans attestations of the better angles over past ones, while neglecting measurements on which their plans charge inadequately. Obviously, the majority of them are a long way from acceptable - either is discovered shy of vital security objectives or absence of basic properties, particularly being screwed over thanks to the security usability strain. To conquer this issue, in this work we first unequivocally characterize a security display that can precisely catch the down to earth capacities. As our principle commitment, another plan is progressed to determine the different issues emerging from client debasement and server trade off, and it is formally demonstrated secure under the harshest foe display up until now. Specifically, by coordinating "honeywords" with a "fluffy verifier", our plan hits "two birds with one stone": it takes out the long-standing security-ease of use struggle that is viewed as obstinate in the writing, while at the same time accomplishing security ensures past the customary ideal security bound. Ash works so far composed are MD5 (128 bits),
\end{abstract}

Index Terms- Two-factor authentication, Online transaction, Password, MPIN, Encryption, Decryption.

\section{INTRODUCTION}

With the quick advancement of conveyed frameworks and the expanding interest for sharing administrations and assets, secure and proficient correspondences between the appropriated user terminals and specialist organizations are raising an ever increasing number of concerns, and it is of absolute significance to ensure the frameworks (e.g., internet business systems [1] and cloud frameworks [2]) and the users' protection and security from malevolent foes. As needs be, user confirmation turns into a fundamental security component for application frameworks to guarantee the realness of the conveying parties. Among the various techniques for user confirmation, secret key verification, MPIN is the most generally utilized and adequate

(C) 2018 Copyright held by the author(s). Published by AIJR Publisher in Proceedings of the $3^{\text {rd }}$ National Conference on Image Processing, Computing, Communication, Networking and Data Analytics (NCICCNDA 2018), April 28, 2018.

This is an open access article under Creative Commons Attribution-NonCommercial 4.0 International (CC BY-NC 4.0) original work is properly cited. ISBN: 978-81-936820-0-5 
component in view of its simple activity, adaptability, similarity and ease points of interest [3], [4]. In such secret word just verification conspires every user is expected to hold a paramount, low-entropy watchword, while the validation server needs to store a watchword related verifier table important to confirm the realness of users.

\section{LITERATURE SURVEY}

Ahmad and Mahmood (2013): Expulsion of cheats in web saving money can't be credited to innovation achievement just, there are different factors as well. In this way, current examine assesses the critical success factors (CSF) for averting and diminishing fakes in web saving money. What's more, these critical success factors reason that saving money inward work force ought to see balance and employer stability to keep away from liberality in cheats in relationship with fraudsters. Correspondingly, another CSF which is additionally upheld by Dr. S. ArumugaPerumal (April 2006) is about presentation of biometric with secret key for fortifying the verification procedure. In addition, administration bolster for assortment of safety efforts like Encryption is another imperative CSF. Besides, not just inward control yet in addition in house interior review is powerful for achievement of web managing an account. In the meantime it is of most extreme significance that clients are made mindful of careful steps to maintain a strategic distance from fakes in web managing an account. Then again if same factors are considered in pessimistic viewpoint like cooperation of interbank staff with fraudsters, phishing, poor confirmation framework, absence of administration bolster, absence of inward review and absence of financial factors, for example, joblessness and neediness, they tend to cause disappointment of web managing an account and it is known as critical failure factors (CFFs). Bashir and Madhavaiah (2014) Determinants of Young Consumers' Intention to Use Internet Banking Services in India: Regardless of whether web managing an account involves standard propensity for nationals of United States of America and United Kingdom and numerous other European nations, yet to the extent Indian economy is concerned still at a blooming level. Howsoever measure of assets be gained by virtual saving money, it won't yield any profits unless embraced by youthful clients. As extremely restricted investigates have been overviewed on youthful clients, this examination has attempted to decide the variables which read youthful clients' brain science in the utilization and acknowledgment of digital keeping money.Research was directed by means of two hundred and fifty understudies of college arranged in Awantipura i.e. Jammu and Kashmir for the surges of Science and Technology. Understudies were chosen on the premise of accommodation testing. Just those youthful understudies who were effective in utilizing PCs were chosen. Another variable presented in this investigation was societal impact which implies found in conduct of a person because of outside or social information sources. For instance learning conveyed to them. Research finished with proposal to enhance the ability of youthful clients for rocker managing

Proceedings of the $3^{\text {rd }}$ National Conference on Image Processing, Computing, Communication, Networking and Data Analytics (NCICCNDA 2018) 
Survey on Two-Factor Authentication with Security in Online Transaction

an account through different ways might be enhanced web engineering, preparing too security techniques and free demos for learning task arrangement of net managing an account.

\section{METHODOLOGY}

\subsection{BASICS OF CRYPTOGRAPHY}

The underlying time of PCs had not seen quite a bit of endeavors put on security as the utilization of PCs was constrained to college and research labs for sharing their information. In the current past, PC organize in the type of Internet has seen a tremendous development in number of hosts associated with it. As of January 2009, the quantity of PCs associated to Internet were assessed at 625,226,456 (625.2 Million) over the world [10]

All the five properties will now be talked about here:

Confidentiality: Otherwise called mystery or protection is about keeping the advantages mystery from the intrusive eyes. The property gives confirmation of keeping unapproved individual from perusing the information. At the end of the day, it gives the confirmation of forestalling revelation of data to unapproved people or frameworks.

Integrity: The property that guarantees the counteractive action of unapproved individual from changing the information. This property is regularly used to accomplish confirmation and non-revocation.

Availability: The property that guarantees an operable and committable condition of a framework or asset constantly. It is more often than not spoken to in view of the Uptime of an asset. Availability is regularly considered as convenience property in light of the fact that if the asset is accessible at that point it is available.

Authenticity: The property that guarantees that the individual, framework or information is bona fide. It includes affirming the legitimacy of a thing who it cases to be.

Non-repudiation: It guarantees that a gathering engaged with an exchange can't revoke or deny the inclusion at a later stage. The affirmation of this property regularly requires the mark and an outsider contribution for lawful approval of the case. To accomplish 'Agony' properties cryptographic systems are utilized.

\subsection{FlowChart outline of framework task}

A flowchart is a sort of graph that speaks to a calculation, work process or process, demonstrating the means as boxes of different sorts, and their request by interfacing them with bolts. This diagrammatic portrayal represents an answer model to a given issue. Flowcharts are utilized as a part of dissecting, outlining, archiving or dealing with a procedure or program in different fields 


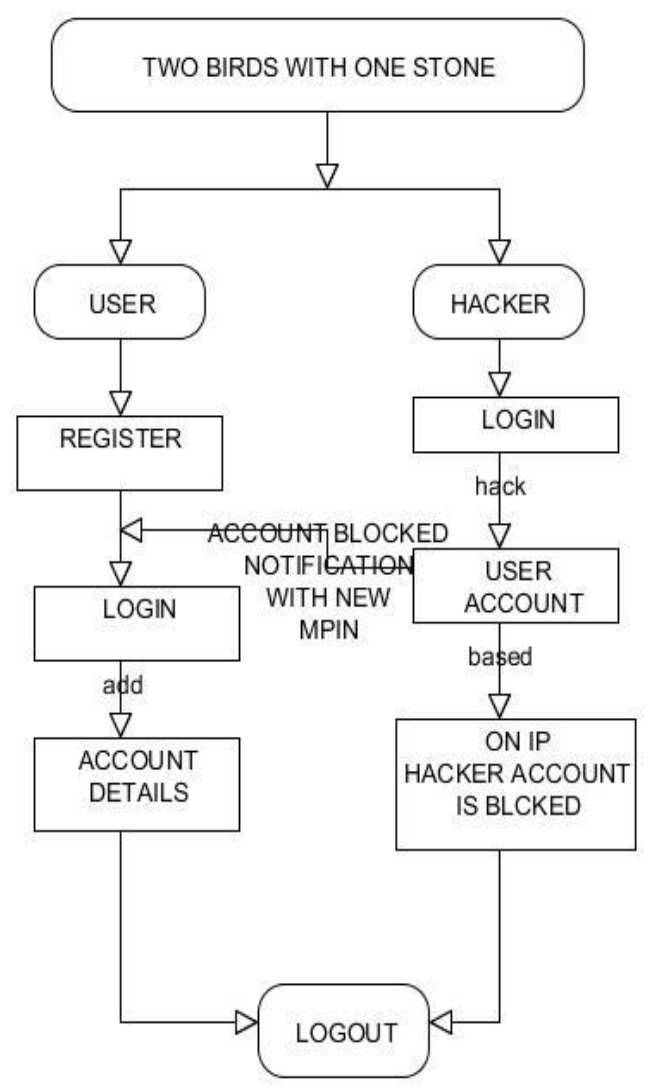

Figure 2.2 Flow chat diagram for user login.

\subsection{MD5 ALGORITHM}

The MD5 hashing calculation is a restricted cryptographic capacity that acknowledges a message of any length as information and returns as yield a settled length process an incentive to be utilized for validating the first message. The MD5 message process hashing calculation forms information in 512-piece squares, separated into 16 words made out of 32 bits each. The yield from MD5 is a 128-piece message process esteem. Calculation of the MD5 process esteem is performed in particular stages that procedure each 512-piece square of information alongside the esteem figured in the former stage: The main stage starts with the message process esteems introduced utilizing back to back hexadecimal numerical qualities. Each stage incorporates four message process passes which control esteems in the present information square and qualities handled from the past piece. The last esteem figured from the last square turns into the MD5 process for that piece Here we used MD5 algorithm to generate a new hash value for each and every transactions made by the user to get a unique id.

Proceedings of the $3^{\text {rd }}$ National Conference on Image Processing, Computing, Communication, Networking and Data Analytics (NCICCNDA 2018) 
Survey on Two-Factor Authentication with Security in Online Transaction

4 REQUIREMENT SPECIFICATION

\subsection{FUNCTIONAL REQUIREMENTS}

An utilitarian necessity archive characterizes the usefulness of a framework or one of its subsystems. It additionally relies on the kind of programming, expected users and the sort of framework where the product is utilized. Useful user necessities might be abnormal state proclamations of what the framework ought to do yet utilitarian framework prerequisites ought to likewise depict plainly about the framework benefits in detail.

\section{MODULES}

There are 3 modules

- Server :Server will block the Hacker

- Hacker:Hacker will login

Hacker will hack the account

- User :User will register and login

User will the account details Hacker

\subsection{SYSTEM ARCHITECTURE}

This system consists of three modules namely user, attacker, and server. Firstly the user registers using his personal credentials which are stored in encrypted format in the sever, at the time of login the user will get MPIN through his mail-id using which he can login in. During the time of transaction the attacker can hack the account and try to get the transactional password which is obtained at the time of transaction to the user. If the user or the attacker inputs wrong password twice the account will be blocked and expires within 2 minutes.

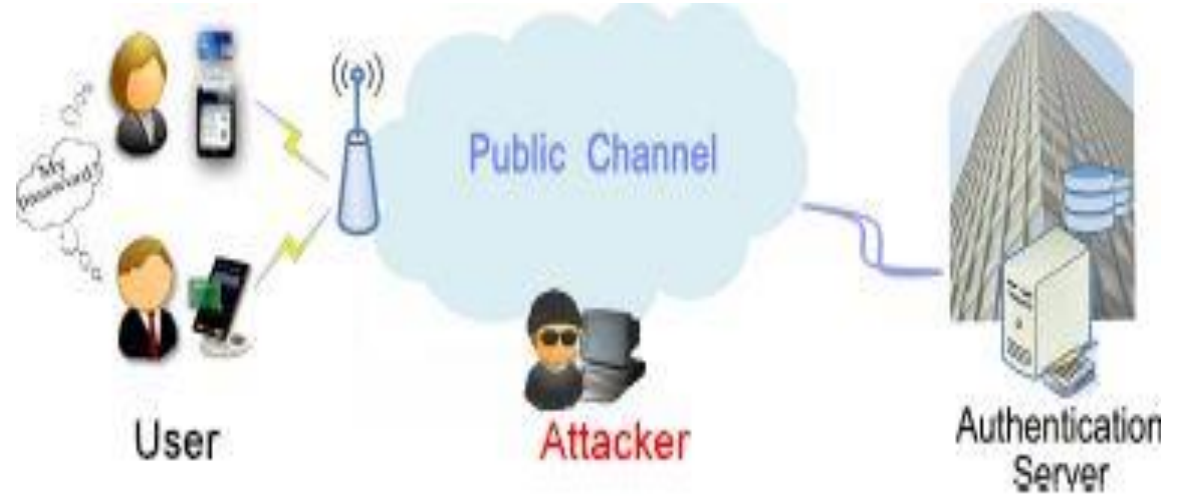

Figure 3.2 System architecture

\section{CONCLUSION}

Here, we have taken a step towards securing the -break-fix-break-fix cycle in the two-factor authentication with security during online transaction in research area. Beyond our proposal of this new scenario which meets practicability, simplicity, and robust notions of security, the proposed system provide a benchmark for the evaluation of current and future two-factor authentication proposals. To the finest of our knowledge. 


\section{FUTURE ENHANCEMENT}

It is expected to help facilitate better assessment of current and future schemes. Our Project is more concrete and comprehensive than related ones and is expected to help facilitate a deeper understanding of the pros and cons of the current and future two-factor schemes. This is of fundamental importance for security engineers to make their choices correctly and for protocol designers to develop practical schemes with better usability-security tradeoffs.

\section{REFERENCES}

[1] J. Bohannon, —Credit card study blows holes in anonymity,ll Science, vol. 347, no. 6221, pp. 467-468, 2015.

[2] M. Nanavati, P. Colp, B. Aiello, and A. Warfield, —Cloud security: A gathering storm,॥Commun. ACM, vol. 57, no. 5, pp. 70-79, 2014.

[3] J. Bonneau, C. Herley, P. Oorschot, and F. Stajano, - The quest to replace passwords: A framework for comparative evaluation of web authentication schemes, $\|$ in Proc. IEEE S\&P 2012, pp. 553-567.

[4] C. Herley and P. Van Oorschot, -A research agenda acknowledging thepersistence of passwords,l IEEE Secur. Priv., vol. 10, no. 1, pp. 28-36, 2012. [5] T. Wu, -The secure remote password protocol,ll in Proc. NDSS 1998. The Internet Society, 1998, pp. 1-15.

[6] J. Katz, R. Ostrovsky, and M. Yung, —Efficient and secure authenticated key exchange using weak passwords, „I J. ACM, vol. 57, no. 1, pp. 1-41, 2009.

[7] M. Abdalla, F. Benhamouda, and P. MacKenzie, - Security of the jpake passwordauthenticated key exchange protocol,॥ in Proc. IEEE S\&P 2015. IEEE Computer Society, 2015, pp. 571-587.

[8] D. Wang, G. Jian, X. Huang, and P. Wang, -Zipf_s law in passwords,॥ Cryptology ePrint Archive, Report 2014/631, pp. 1-33, 2014, http:// eprint.iacr.org/2014/631.pdf.

[9] M. Adeptus, Hashdumps and Passwords, May 2014, http://www. adeptusmechanicus.com/codex/hashpass/hashpass.php.

[10] J. Ma, W. Yang, M. Luo, and N. Li, -A study of probabilistic password models, in Proc. IEEE S\&P 2014. IEEE, 2014, pp. 538-552. 